\title{
Risk of Transfusion-Transmitted Hepatitis E Virus: Correspondence
}

\author{
Rujittika Mungmunpuntipantip ${ }^{1} \cdot$ Viroj Wiwanitkit $^{2}$
}

Received: 1 February 2022/Accepted: 17 February 2022/Published online: 3 March 2022

(C) Indian Society of Hematology and Blood Transfusion 2022

Dear Editor, we would like to comment on the publication "The Risk of Transfusion-Transmitted Hepatitis E Virus: Evidence from Seroprevalence Screening of Blood Donations [1]." Wong et al. concluded that "The higher rates of HEV infection in blood from older donors and donors who are non-professional workers may provide insights into targeted groups for blood screening [1]." We agree that the estimation of risk for the transmission of HEV via blood transfusion is interesting. The present study reported on the results from serological screening. The diagnostic property of the serological test is a major concern. Because the diagnostic serological test may have limitations, the true HEV contamination rate in donated blood samples may differ from the rate reported by Wong et al.

There could be a number of samples that test positive for HEV contamination but turn out to be false positives. According to a recent Qatari study, the Wantai HEV IgG assay could yield $32.1 \%$ of positive screening results [2]. When the RT-PCR test was used, the rate was $23.1 \%$ [2]. Many non-infectious PCR negative samples are reported as positive results by the HEV IgG assay. In addition, when compared to other types of test kits, the Wantai HEV IgG assay employed in this study has a poorer specificity for determining HEV infection [3]. This indicates that the WAntai HEV IgG assay can produce a significant proportion of false positive results. As a result, the current HEV contamination rate observed in this report should be higher than the actual rate. As a result, it can be demonstrated that the seroprevalence rate may result in an overestimation of risk.

\section{Declarations}

Conflict of interest None.

\section{References}

1. Wong LP, Lee HY, Khor CS et al (2022) The risk of transfusiontransmitted hepatitis E virus: evidence from seroprevalence screening of blood donations. Indian J Hematol Blood Transfus 38:145-152. https://doi.org/10.1007/s12288-021-01428-7

2. Al Absi ES, Al-Sadeq DW, Khalili M, Younes N, Al-Dewik N, Abdelghany SK, Abouzid SS, Al Thani AA, Yassine HM, Coyle PV, Nasrallah GK (2021) The prevalence of HEV among non-A-C hepatitis in Qatar and efficiency of serological markers for the diagnosis of hepatitis E. BMC Gastroenterol 21(1):266

3. Zhang Q, Zong X, Li D, Lin J, Li L (2020) Performance evaluation of different commercial serological kits for diagnosis of acute hepatitis E viral infection. Pol J Microbiol 69(2):217-222

Publisher's Note Springer Nature remains neutral with regard to jurisdictional claims in published maps and institutional affiliations.
Rujittika Mungmunpuntipantip rujittika@gmail.com

1 Bangkok, Thailand

2 Dr DY Patil University, Pune, India 